Physics In ColLision - Stanford, California, June 20-22, 2002

\title{
LARGE BULK MATTER SEARCH FOR FRACTIONAL CHARGE PARTICLES
}

\author{
Irwin Lee \\ Stanford Linear Accelerator Center
}

\begin{abstract}
We have carried out the largest search for stable particles with fractional electric charge, based on an oil drop method that incorporates a horizontal electric field and upward air flow. No evidence for such particles was found, giving a 95\% C.L. upper limit of $1.15 \times 10^{-22}$ particles per nucleon on the abundance of fractional charge particles in silicone oil for $0.18 e \leq\left|Q_{\text {residual }}\right| \leq 0.82 e$.
\end{abstract}

\section{Introduction}

We have carried out the largest search for fractional electric charge elementary particles in bulk matter using $70.1 \mathrm{mg}$ of silicone oil. That is, we looked for stable particles whose charge $Q$ deviates from $N e$ where $N$ is an integer, and $e$ is the electron charge. No evidence for such particles was found. We used our new version [1] of the Millikan oil drop method containing two innovations compared to the classical method that we used in Halyo et al. [2]. One innovation is that the drop charge is obtained by observing the drop motion in a horizontal, alternating electric field compared to the classical use of a vertical electric field. The other innovation is the use of an upward flow of air to reduce the vertical terminal velocity of the drop, which enabled us to use larger drops, about $20.6 \mu \mathrm{m}$ in diameter compared to the $10 \mu \mathrm{m}$ drops used in our previous experiments. Figure 1 depicts the principle of the technique. 


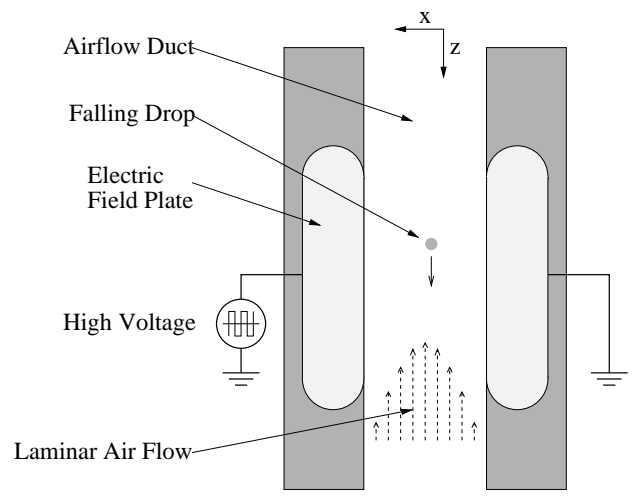

Figure 1: The experimental principle behind the search technique.

\section{Apparatus}

Recent advances in inkjet printing technology and computer imaging are the key enabling technologies behind the experiment. The apparatus is built around a micromachined [3], drop-on-demand fluid ejector, and a digital charge-coupled device (CCD) camera. The drop ejector produces extremely uniform oil drops with radii constant to $\pm 0.2 \%$. This ability to precisely control the drop radius, in addition to the large statistics gained by measuring many drops, allows the experiment to be self-calibrating, significantly reducing the possibility of an artifact.

The experiment is completely automated. The measurement region, approximately $2.3 \mathrm{~mm}$ horizontally by $3.0 \mathrm{~mm}$ vertically, is viewed by the CCD camera producing a $10 \mathrm{~Hz}$ stream of images in 8 bit greyscale at $736 \times 240$ pixel resolution. This data is acquired, stored, and analyzed in real-time using custom written software. This software analyzes the images, measuring the positions of the drops to sub-pixel accuracy, and performs pattern recognition to identify the trajectories of individual drops. The charge $Q$ on each drop is calculated from the best possible fit to the measured trajectory, where the precision is primarily limited by Brownian motion.

\section{Results}

Data was collected for 30 weeks. From this sample, approximately $11 \%$ of the drops were rejected using predetermined selection criteria. These selection criteria eliminated incorrectly measured drops, and were unbiased with regard to fractional charge. A total of $17 \times 10^{6}$ drops were accepted into the final data sample. 
The resulting distributions of $Q$ and the residual charge $Q_{r}$, defined to be $Q_{r}=Q-N_{l} e$ where $N_{l}$ is the largest integer less than $Q / e$, are shown in Figure 2. No drops with fractional charge were observed, yielding a $95 \%$ confidence level upper limit on the abundance of fractional charge particles in silicone oil, for $0.18 e \leq Q_{r} \leq 0.82 e$, of $1.17 \times 10^{-22}$ particles per nucleon. This result is described in detail in Lee et al. . $\mid$.
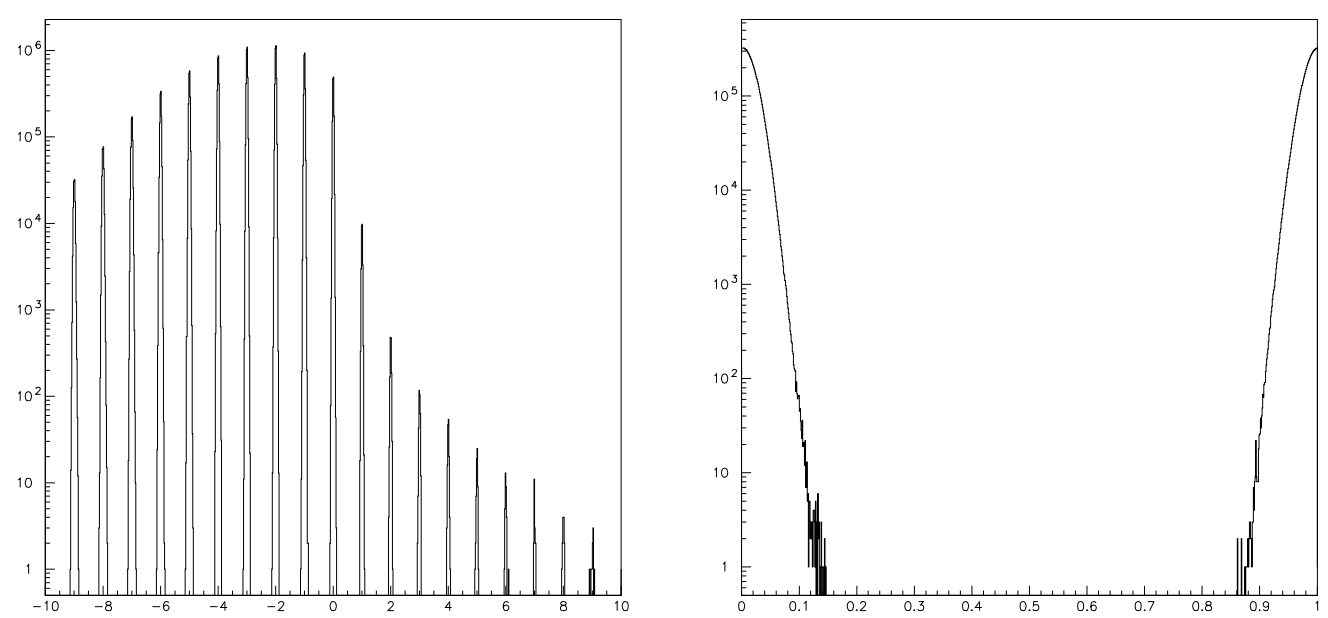

Figure 2: The overall charge distribution (left), in units of e, of the $17 \times 10^{6}$ oil drops studied and the residual charge $Q_{r}$ (right).

\section{Acknowledgments}

This experiment was performed at SLAC under the direction of Martin Perl, and in collaboration with Sewan Fan, Valerie Halyo, Peter C. Kim, Eric R. Lee, Dinesh Loomba, and Howard Rogers. Thanks also to Klaus S. Lackner and Gordon Shaw. This work was supported by Department of Energy contract DE-AC03$76 \mathrm{SF} 00515$.

\section{References}

1. D. Loomba et al., Rev. Sci. Instrum. 71, 3409 (2000).

2. V. Halyo et al., Phys. Rev. Lett. 84, 2576 (2000).

3. E. R. Lee and M. L. Perl, U.S. Patent 5,943,075, Aug. 24, 1999

4. I. T. Lee et al., Phys. Rev. D 66, 012002 (2002). 